Supporting Information for

\title{
Experimental and numerical investigation of flow
}

\section{field and soot particle size distribution of methane-}

containing gas mixtures in a swirling burner

Zari Musavi $\neq^{\Psi}$, Yao Zhang $\neq^{\Lambda}$, Etienne Robert ${ }^{4}$ and Klas Engvall ${ }^{\Psi}$

${ }^{\Psi}$ KTH Royal Institute of Technology, Dept. of Chemical Engineering, Stockholm, Sweden

${ }^{\wedge}$ Polytechnique Montréal, Dept. of Mechanical Engineering, Montréal, Canada

Supportive information includes the following contents:

Figure S1. Comparison of mean velocity contour results between a) experimental; and three

turbulent model alternatives: b) transition SST model; c) RNG k-epsilon; and d) RSM.

Table S1. Detailed settings of all boundaries for the numerical model 
Table S2. Summary of reactive model settings for the numerical model
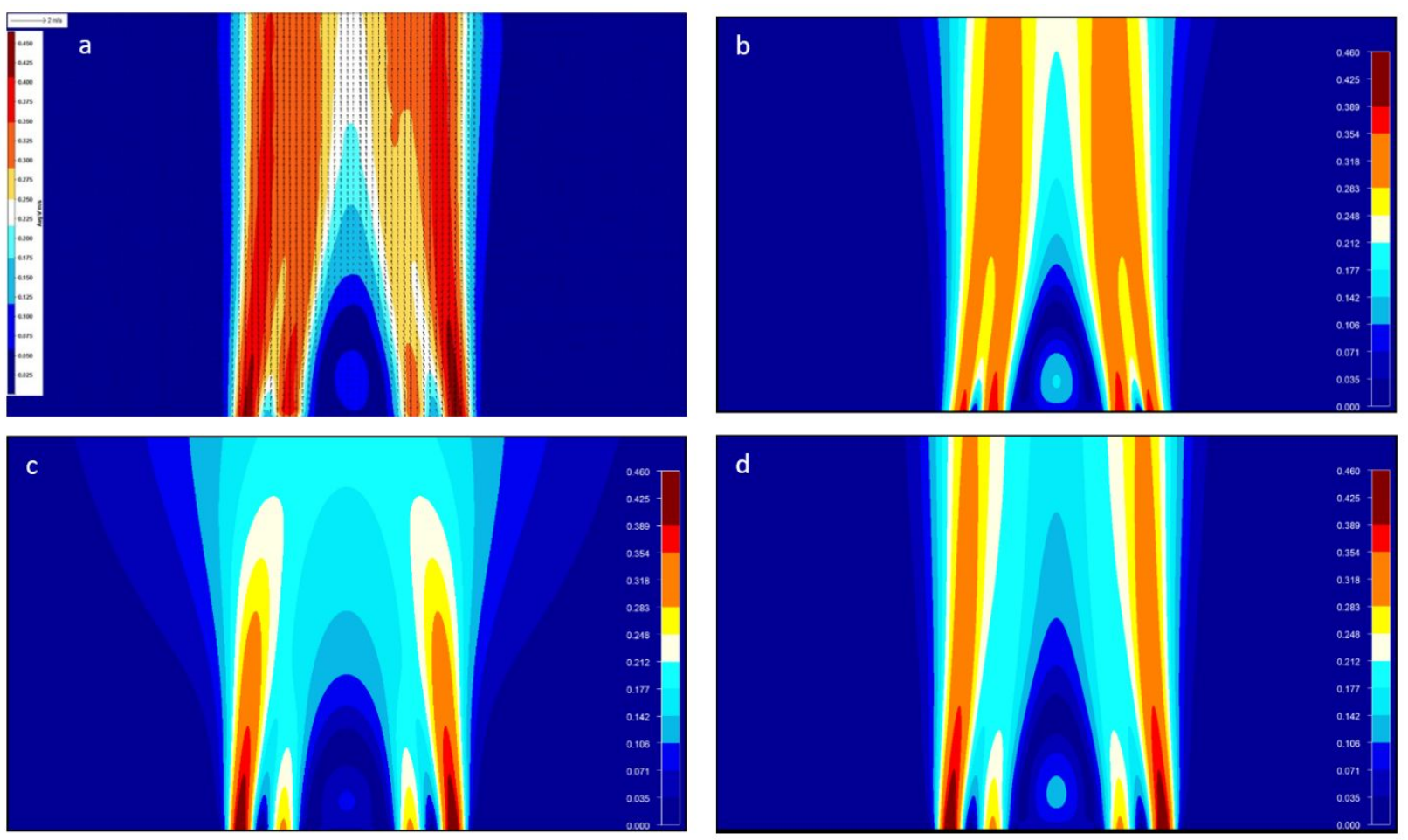

Figure S1. Comparison of mean velocity contour results between a) experimental; and three

turbulent model alternatives: b) transition SST model; c) RNG k-epsilon; and d) RSM. 
Table S1. Detailed settings of all boundaries for the numerical model

\begin{tabular}{|c|c|c|c|c|c|c|c|c|c|c|c|c|c|c|}
\hline NAME & A & B & $\mathrm{C}$ & D & E & $\mathrm{F}$ & G & $\mathrm{H}$ & I & $I^{\prime}$ & $\mathrm{J}$ & K & $\mathrm{L}$ & $\mathrm{M}$ \\
\hline $\begin{array}{l}\text { Boundary } \\
\text { type }\end{array}$ & wall & $\begin{array}{l}\text { veloc } \\
\text { ity- } \\
\text { inlet }\end{array}$ & wall & $\begin{array}{l}\text { veloc } \\
\text { ity- } \\
\text { inlet }\end{array}$ & wall & wall & wall & wall & wall & wall & wall & wall & $\begin{array}{l}\text { outfl } \\
\text { ow }\end{array}$ & axis \\
\hline $\begin{array}{l}\text { Wall } \\
\text { Thickness, } \\
\text { mm }\end{array}$ & & & & & & & 24.5 & 24.5 & & & & & & \\
\hline $\begin{array}{l}\text { Material } \\
\text { Name }\end{array}$ & steel & & steel & & steel & steel & steel & steel & quartz & quartz & quartz & quartz & & \\
\hline $\begin{array}{l}\text { Wall } \\
\text { Roughness } \\
\text { Height (m) }\end{array}$ & 0 & & 0 & & 0 & 0 & 0 & & 0 & & & & & \\
\hline $\begin{array}{l}\text { Wall } \\
\text { Roughness } \\
\text { Constant }\end{array}$ & 0.5 & & 0.5 & & 0.5 & 0.5 & 0.5 & & 0.5 & & & & & \\
\hline $\begin{array}{l}\text { Thermal BC } \\
\text { Type }\end{array}$ & 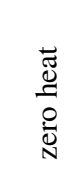 & 言 & $\begin{array}{l}\overrightarrow{\mathbb{J}} \\
\stackrel{\Xi}{0} \\
\stackrel{0}{0} \\
\text { N }\end{array}$ & 宅 & 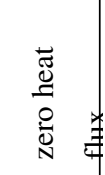 & 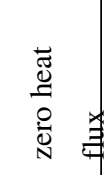 & 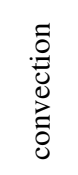 & 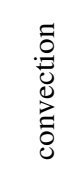 & 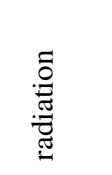 & 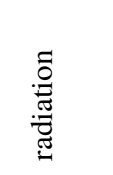 & 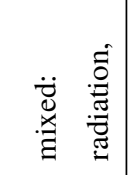 & $\begin{array}{l}\tilde{0} \\
\stackrel{0}{0} \\
\stackrel{0}{0} \\
0 \\
0\end{array}$ & & \\
\hline $\begin{array}{l}\text { Convective } \\
\text { Heat } \\
\text { Transfer } \\
\text { Coefficient, } \\
\text { W/m-K }\end{array}$ & & & & & & & 4 & 4 & & & 8 & 8 & & \\
\hline $\begin{array}{l}\text { Free Stream } \\
\text { Temperature }\end{array}$ & & & & & & & 293 & 293 & & & 293 & 293 & & \\
\hline $\begin{array}{l}\text { Radiation } \\
\text { BC Type }\end{array}$ & $\begin{array}{l}\stackrel{\mathscr{z}}{\tilde{z}} \\
\stackrel{\tilde{o}}{0}\end{array}$ & & $\begin{array}{l}\stackrel{\Xi}{\tilde{\Xi}} \\
\text { ŏ }\end{array}$ & & $\begin{array}{l}\stackrel{0}{\tilde{g}} \\
\stackrel{\vec{g}}{\sigma}\end{array}$ & $\begin{array}{l}\frac{0}{\tilde{J}} \\
\text { ôّ }\end{array}$ & 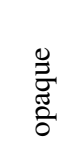 & 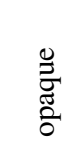 & 光 & 峁 & 䓫 & 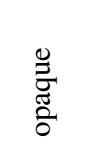 & & \\
\hline $\begin{array}{l}\text { Internal } \\
\text { emissivity } \\
\text { for each } \\
\text { band }(3\end{array}$ & $\begin{array}{l}(0.4 \\
0.4 \\
0.4)\end{array}$ & 0 & $\begin{array}{l}(0.4 \\
0.4 \\
0.4)\end{array}$ & 0 & $\begin{array}{l}(0.4 \\
0.4 \\
0.4)\end{array}$ & $\begin{array}{l}(0.4 \\
0.4 \\
0.4)\end{array}$ & $\begin{array}{l}(0.4 \\
0.4 \\
0.4)\end{array}$ & $\begin{array}{l}\left(\begin{array}{ll}0 & 0 \\
0)\end{array}\right.\end{array}$ & $\left(\begin{array}{lll}0 & 0 & 0\end{array}\right)$ & $\left(\begin{array}{lll}0 & 0 & 0\end{array}\right)$ & $\left(\begin{array}{lll}0 & 0 & 0\end{array}\right)$ & $\left(\begin{array}{lll}0 & 0 & 0\end{array}\right)$ & 0 & \\
\hline
\end{tabular}




\begin{tabular}{|c|c|c|c|c|c|c|c|c|c|c|}
\hline $\begin{array}{l}\text { bands are } \\
\text { defined)- }\end{array}$ & & & & & & & & & & \\
\hline $\begin{array}{l}\text { Diffuse } \\
\text { fraction for } \\
\text { each band }\end{array}$ & $\begin{array}{l}(11 \\
1)\end{array}$ & $\begin{array}{l}(11 \\
1)\end{array}$ & $\begin{array}{l}(11 \\
1)\end{array}$ & $\begin{array}{l}(11 \\
1)\end{array}$ & $\begin{array}{l}\left(\begin{array}{ll}1 & 1 \\
1\end{array}\right)\end{array}$ & $\begin{array}{l}(11 \\
1)\end{array}$ & $\left(\begin{array}{lll}1 & 1 & 1\end{array}\right)$ & $\left(\begin{array}{lll}1 & 1 & 1\end{array}\right)$ & $\left(\begin{array}{lll}1 & 1 & 1\end{array}\right)$ & $\left(\begin{array}{lll}1 & 1 & 1\end{array}\right)$ \\
\hline $\begin{array}{l}\text { External } \\
\text { Emissivity }\end{array}$ & & & & & & & & & 0.01 & \\
\hline $\begin{array}{l}\text { External } \\
\text { Radiation } \\
\text { Temperature }\end{array}$ & & & & & & & & & 293 & \\
\hline
\end{tabular}

Table S2. Summary of reactive model settings for the numerical model

\begin{tabular}{|c|c|}
\hline Model & Settings \\
\hline Space & Axisymmetric swirl \\
\hline Time & Steady \\
\hline Viscous & Transition SST model \\
\hline Radiation & Discrete ordinates, 3 non-gray bands \\
\hline Species & 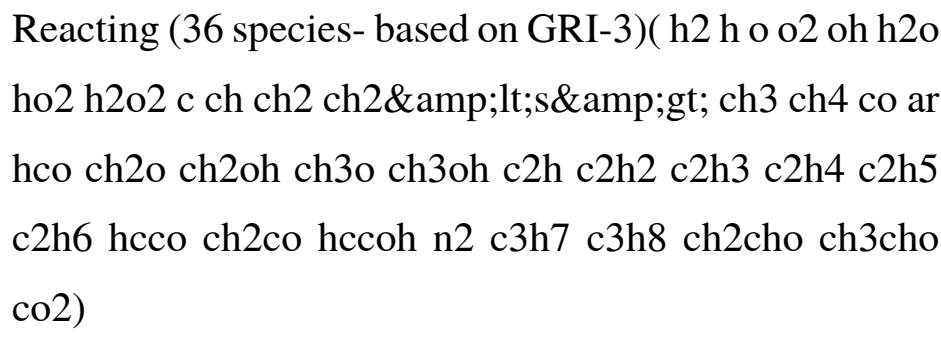 \\
\hline Soot & $\begin{array}{l}\text { Nucleation based on acetylene, surface growth based on } \\
\text { HACA mechanism, oxidation by oxygen and hydroxyl } \\
\text { radical }\end{array}$ \\
\hline
\end{tabular}




\begin{tabular}{ll} 
Discretization Scheme for & Scheme \\
different variables & \\
\hline Pressure & PRESTO! \\
Momentum & Second Order Upwind \\
Swirl Velocity & Second Order Upwind \\
Turbulent Kinetic Energy & Second Order Upwind \\
Specific Dissipation Rate & Second Order Upwind \\
Intermittency & Second Order Upwind \\
Momentum Thickness Re & Second Order Upwind \\
Species & Second Order Upwind \\
Soot Method of Moments & Second Order Upwind \\
Energy & Second Order Upwind
\end{tabular}

